\title{
On the detection of signals embedded in natural scenes
}

\author{
TERRY CAELLI and GIAMPAOLO MORAGLIA \\ University of Alberta, Edmonton, Alberta, Canada
}

\begin{abstract}
In this paper we consider the processes by which observers can detect and recognize signals embedded in natural scenes and images. Although our results do not strongly support detection processes based strictly upon the cross-correlation (template-matching) of the signal and image luminance profiles, they do support a version of cross-correlation based upon the comparison of their "structural similarities." This latter correlational measure may be separated from the energy component in the cross-correlation function. Of particular importance to the structural similarity between the signal and image is their characteristic edge components, and we also show that recognition performance was consistent with the cross-correlations between the signal and image edge-only versions. This finding, again, exemplifies the importance, for the analysis of pattern detection and recognition, of isolating structural components from energy detection processes per se.
\end{abstract}

Although the task of detecting a signal embedded in an image is a common one for the human visual system, the spatial determinants of such a task are rarely studied, particularly with naturally occurring images. Exceptions to this are the investigations of Julesz $(1981,1985$; Julesz \& Bergan, 1983), who studied the conditions for detecting specified micropatterns in textures, and Burgess and Ghandeharian (1984a, 1984b), who determined the efficiency of observers in detecting signals embedded in noise.

According to Julesz, such "attentional" processes are dependent on the ability of the human visual system to differentiate among specific spatial codes (e.g., orientation, aspect ratios, etc.), whereas, for Burgess and Ghandeharian, detection of signals in noise is adequately determined by simple cross-correlation, or matched filtering, between the signal and signal-plus-noise image. Although it seems obvious that simple energy-detection models, based on the direct perceptual cross-correlation of the input luminance profiles, are inadequate in attempting to explain all types of pattern recognition behavior, some form of correlation seems necessary for any reasonably broad model for this task. It is therefore of central interest to this study to consider the type of spatial information that is cross-correlated in such tasks when more natural images are used.

According to Burgess and Ghandeharian's (1984a, 1984b) formulation, the ideal observer, using a maximum

This project was funded by Grant A2568 from the Natural Sciences and Engineering Research Council of Canada and by Grant RA2525 from the Alberta Heritage Foundation for Medical Research. We wish to thank Marc Green for the many discussions that have led to the completion of this paper. The authors' mailing address is: Department of Psychology, University of Alberta, Edmonton, Alberta, Canada T6G 2E9. a posteriori probability (MAP) decision strategy, determines the cross-correlation between the expected signal and the image data at each of $M$ locations. Each correlation result is weighted according to the probability of the signal's being present at that location $(1 / M$ for the uniform case). The optimum decision strategy is then to select the location with the highest weighted cross-correlation value (Burgess \& Ghandeharian, 1984b, p. 907).

This formulation predicts that the probability of correct detection is monotonic with the signal-to-noise (background) energy ratio, which increases with the correlation between signal and signal-plus-noise. Also, false alarms are likely to occur, according to the MAP strategy, when the correlation between signal and noise (image) alone exceeds that of the signal-plus-noise case or a given threshold value. Employing simple signals (a disk, a square, and a two-cycle sine wave) and spectrally flat broadband noise, Burgess and Ghandeharian (1984b) not only demonstrated results consistent with this decision model, but also ascertained the detection efficiency to be at about $50 \%$ of the ideal detector.

The aim of this paper is to consider experimental and theoretical extensions of their work to include the detection of signals in more naturally occurring images, and also to consider some initial aspects of the spatial coding and correlation mechanisms that may be operating in this complex (nonstationary) situation. In this way, some connection may be established between the two abovementioned approaches.

Although it seems that the ability of humans to detect signals embedded in homogeneous (stationary) white noise can be predicted by direct cross-correlation and an ideal detector signal-to-noise ratio mechanism, this strategy does not work well in nonwhite images, or in natural scenes, for the following reasons. Define the signal and embedding image as $S(x, y)$ and $I(x, y)$, respectively, where 
$(x, y)$ refer to a locally Euclidean retinotopic coordinate system envisaged to be no greater than $4^{\circ}$ in aperture. The cross-correlation function between $S$ and $S+I\left(I_{s}\right)$ is defined by:

$$
C(x, y)=\int_{-\infty}^{\infty} \int_{\infty} S(\alpha, \beta) I_{s}(x+\alpha, y+\beta) d \alpha d \beta
$$

or,

$$
C=S^{*} * I_{s}=S^{*}{ }_{*} S+S^{*} * I,
$$

where $I_{s}(x, y)=S(x, y)+I(x, y)$ for $S^{*}$ denoting the complex conjugate of $S$ and $*$ denoting convolution. Equation 2 shows that $C$ reduces to the signal autocorrelation function and the signal to image cross-correlation function.

One problem of matching based on this direct form of cross-correlation is that of false alarms. According to the Cauchy Schwarz inequality, the cross-correlation function peaks when $S+I \equiv \lambda S$, that is:

$$
\begin{aligned}
& \int_{-\infty}^{\infty} \int_{\infty} S(\alpha, \beta) I_{s}(x+\alpha, y+\beta) d \alpha d \beta \\
& \leq \sqrt{\int_{-\infty}^{\infty} \int^{2} S^{2}(\alpha, \beta) \mathrm{d} \alpha \mathrm{d} \beta \cdot \int_{-\infty}^{\infty} \int_{s}^{2}(x+\alpha, y+\beta) d \alpha d \beta} .
\end{aligned}
$$

When the signal is always added to the background, the energy present in the specific location of the signal will most likely be the maximum over the full image. However, in normal pattern detection situations this may not be the case because the signal to be detected is an integral part of the image. In this latter case there could be many regions in $I_{s}(x, y)$ in which the cross-correlator varies as a function of the background energy factor,

$$
\int_{-\infty}^{\infty} \int_{s}^{2}(x+\alpha, y+\beta) d \alpha d \beta
$$

and is usually corrected, in digital image processing, by the normalization of Equation 3 as:

$$
\frac{\int_{-\infty}^{\infty} \int_{-\infty} S(\alpha, \beta) \cdot I_{s}(x+\alpha, y+\beta) d \alpha d \beta}{\sqrt{\int_{-\infty}^{\infty} \int_{s}^{2}(x+\alpha, y+\beta) d \alpha d \beta}} \leq \int_{-\infty}^{\infty} \int_{-\infty} S^{2}(\alpha, \beta) d \alpha d \beta=E_{s}
$$

This cross-correlation function is now upper-bounded by the signal energy $\left(E_{s}\right)$. The direct cross-correlator, therefore, produces a large number of signal candidates, only one of which is the correct response. Such cases are illustrated in Figure 1-the signals and images used in our experiment. Here the signals (two-dimensional Gabor signals, see below) were embedded in the images at the center $\left(x_{0}, y_{0}\right)$ according to:

$$
\begin{aligned}
& I_{s}\left(x-x_{0}, y-y_{0}\right) \\
& \quad=I\left(x-x_{0}, y-y_{0}\right)+S\left(x-x_{0}, y-y_{0}\right)-\bar{S},
\end{aligned}
$$

where $\bar{S}$ corresponded to the space-average luminance of the signal. In other words, the average luminance within the neighborhood of $\left(x_{0}, y_{0}\right)$ in the image was not altered after addition of the signal. The visibility of each signal shown in Figure 1 is determined by a number of factors, not excluding the energy of $I_{s}$, but, as will be seen, not totally explained by this factor.

A number of previous authors have discussed the falsealarm problem associated with pattern recognition or matching by cross-correlation (e.g., Papoulis, 1968; Rosenfeld \& Kak, 1982), and it is apparent that some form of indirect cross-correlation is more appropriate. That is, rather than the direct cross-correlation between $S$ and $S+I\left(I_{s}\right)$, the idea here is that both $S$ and $I$ are decomposed into outputs of separate spatial analyzers. These separate "views" of $S$ and $S+I$ are then cross-correlated and, either by summation or selection, a decision is reached from combining the outputs of each subdetector. One particular example of this model is the "derivative matched filter," where both $S$ and $S+I$ are differentiated by at least one bandpass filter. An example of this would be the cross-correlation of a variety of edge-only versions of $S$ and $I_{s}$.

Viewed as a system, the properties of the point-spread function for each spatial analyzer (filter) could well fit those of spatial-frequency channels, or perceptive fields, as described recently by Caelli and Hübner (1983), Daugman $(1980,1983)$, and Wilson and Gelb (1984). However, the problems with such a priori definitions of the decomposition rule are that (1) they may not actually reflect the underlying detector structures for human vision, and (2) their lack of adaptability may well bias any comparison between the direct and indirect correlation models. In an attempt to minimize these problems, we have used signals whose profiles are similar to those corresponding to candidate spatial-frequency-channel pointspread functions at a given orientation.

The (target) signals shown in Figure 1a are twodimensional Gabor signals, being bivariate Gaussian windows with a sinusoidal modulation of the equal-variance form (Daugman, 1980, 1983; Papoulis, 1968):

$$
\begin{aligned}
S(x, y)= & e^{-\left(\left(x-x_{0}\right)^{2} / a^{2}+\left(y-y_{0}\right)^{2} / b^{2}\right)} \\
& \cdot \mathrm{e}^{-2 x i\left(u_{0}\left(x-x_{0}\right)+v_{0}\left(y-y_{0}\right)\right)} ; \quad a=b
\end{aligned}
$$

where $\left(x_{0}, y_{0}\right)$ and $\left(u_{0}, v_{0}\right)$ correspond to their centers in the image and two-dimensional frequency domains, respectively. These signals are localized in the spectrum since the Fourier transform of Equation 5 is:

$$
\begin{aligned}
\mathscr{F}(S(x, y))= & e^{-\left(\left(u-u_{0}\right)^{2} a^{2}+\left(v-v_{0}\right)^{2} b^{2}\right)} \\
& \mathrm{e}^{2 \pi i\left(x_{0}\left(u-u_{0}\right)+y_{0}\left(v-v_{0}\right)\right)} ; \mathrm{a}=\mathrm{b},
\end{aligned}
$$




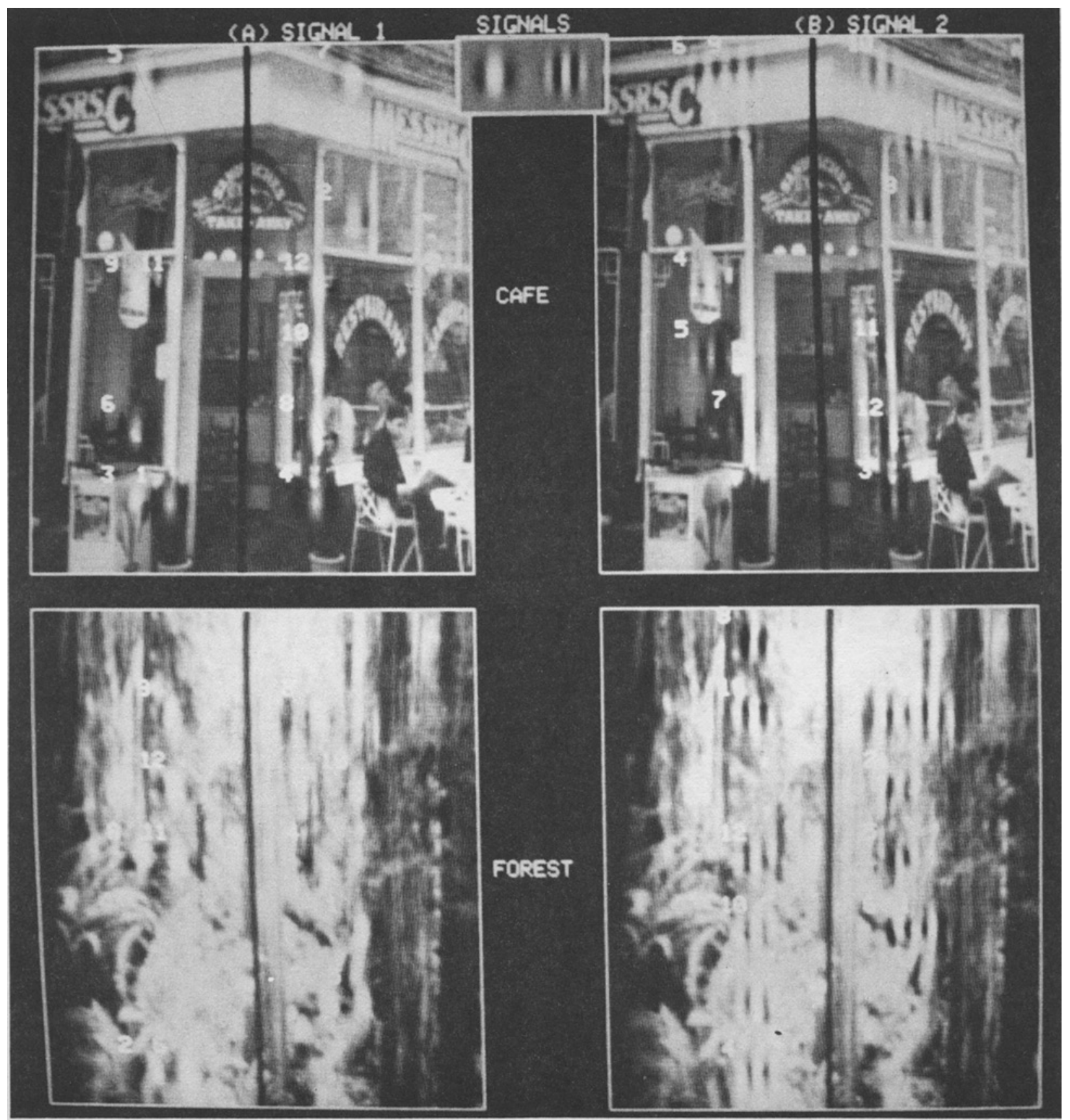

Figure 1. Top center: Two signals used in the experiment. They were added to each of the 12 positions (per image) after stripping the DC (space average luminance) off the signal. The center line was used to denote left and right regions for the two-alternate falsechoice task.

where $a$ corresponds to the space constant [value of

$$
r=\sqrt{\left(x-x_{0}\right)^{2}+\left(y-y_{0}\right)^{2}}
$$

at which the Gaussian decays to $1 / e(\cong .37)$ and $(u, v)$ to the frequency domain coordinate system, measured in cycles per degree (cpd)].

The aim of the following experiment was to determine the detectability of these signals embedded in the images shown in Figure 1 and to investigate to what extent cross- correlation models predict both detection and recognition behavior.

\section{METHOD}

\section{Stimulus and Apparatus}

All signals and images were generated and displayed (via an ITI image processing system coupled to a PDP-11/23 computer) on an Electrohome 14-in. TV monitor with P32 phosphor. The space average luminance was always fixed at $30 \mathrm{~cd} / \mathrm{m}^{2}$, and contrast, at $80 \%$ 


\section{FOREST}

(A)

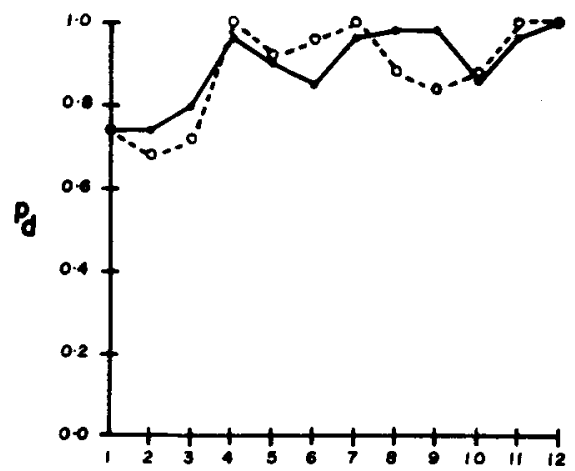

(s)

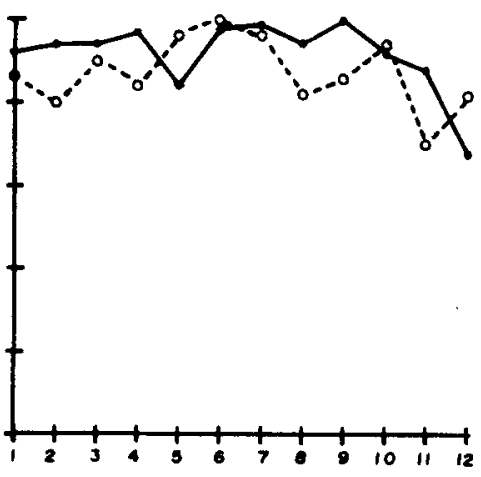

POSITION

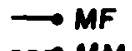

\section{CAFE}

(c)

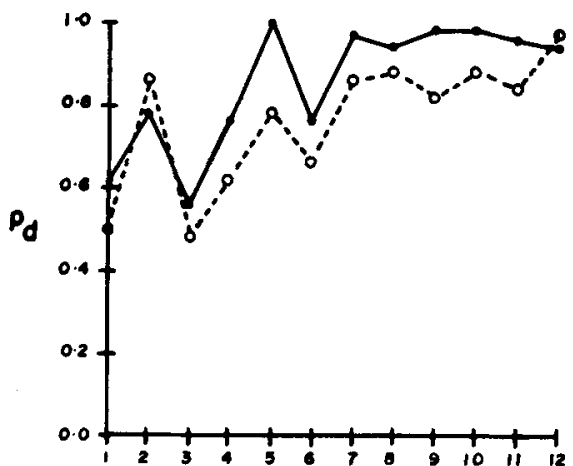

(0)

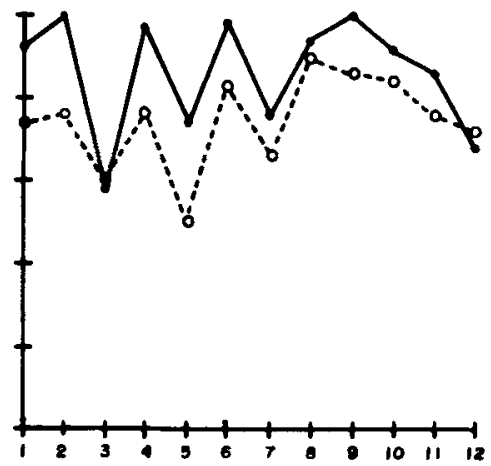

POSITION

Figure 2. Detection probabilities $\left(P_{d}\right)$ as a function of the two images, signals, and positions, for Observers M.F. and M.M. (A) and (C) present the results for Signal 1; (B) and (D) do so for Signal 2. Signals and image positions are displayed in Figure 1.

$\left[\left(l_{\max }-l_{\min } / l_{\max }+l_{\min }\right) \times 100, l=\right.$ luminance $]$. The two signals were generated on a $32 \times 32$ pixel format consisting of an 8-pixel space constant (decay to 1/ $e$ value for the bivariate Gaussian and horizontal cosine frequency modulations of 16 and 8 pixels per cycle (Figure 1a, Equation 5). The images (forest and cafe, Figure 1) had a $256 \times 256$, 8-bit format with a black line down the center, and were used in a four-alternative forced-choice paradigm. On any given trial, the signal was added to one of 12 positions ( 6 on each side of the black line), as shown in Figure 1 and according to Equation 4. This method produced no change in space average luminance in the area, since the average luminance was subtracted from the signal before being added to the background. This resulted in a true "embedding," or mixing, of the signal with the background image, and made it impossible for detection to be based on either peak detection or average luminance differences alone.

The images were displayed in a $2.8^{\circ}$ aperture. The signals subtended a visual angle of $21^{\prime}$, resulting in frequency modulations of 5.7 and $11.4 \mathrm{cpd}$ for the 16 and 8 pixels per cycle values, respectively. The observer was seated $1.75 \mathrm{~m}$ from the screen with his head resting on a chinrest. Responses were made using a four-button detection/recognition paradigm.

\section{Subjects and Procedure}

Two naive observers with normal acuity served in this study. Prior to the start of the experiment, the observers were allowed free inspection of the background images (without embedded signals) in optimal viewing conditions. Familiarity with these images was deemed to be helpful in minimizing the role of practice effects arising from the repeated presentation of the backgrounds during the test runs. The task consisted of a four-alternative forced choice of the following kind. On every trial, one of the two signals was displayed $(p=.5)$ in the center of the viewing aperture for $1 \mathrm{sec}$. After a 300msec interval, the background image was presented for $200 \mathrm{msec}$ with a signal embedded in one of the 12 positions illustrated in 


\section{FOREST}

(A)

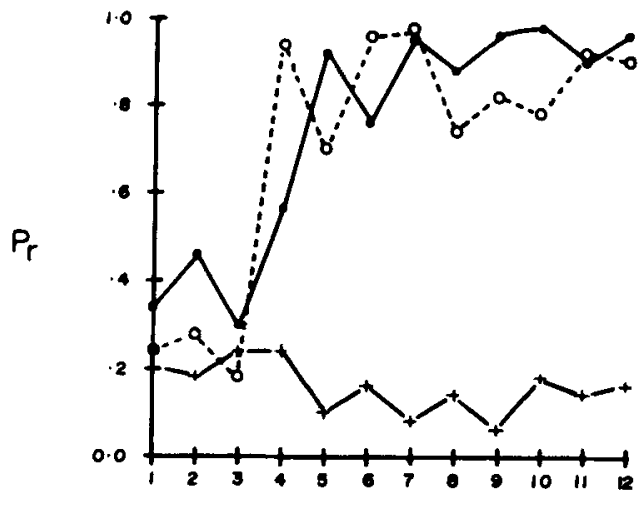

(B)

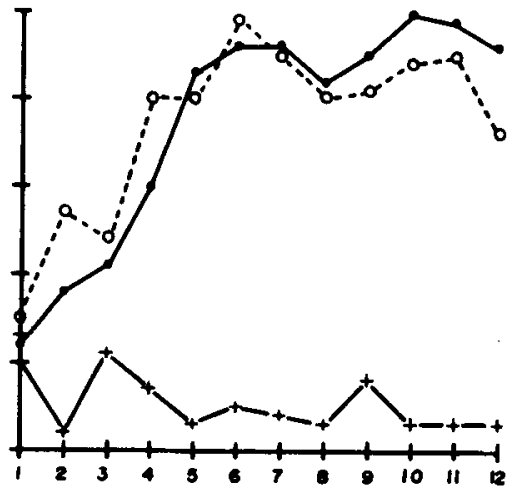

\section{CAFE}

(c)

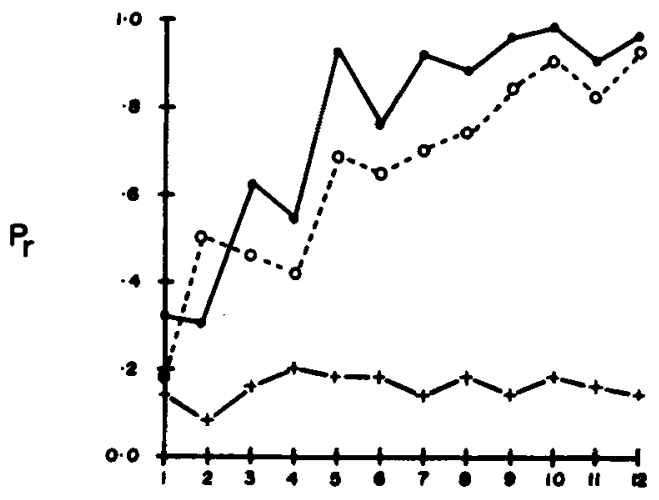

(D)

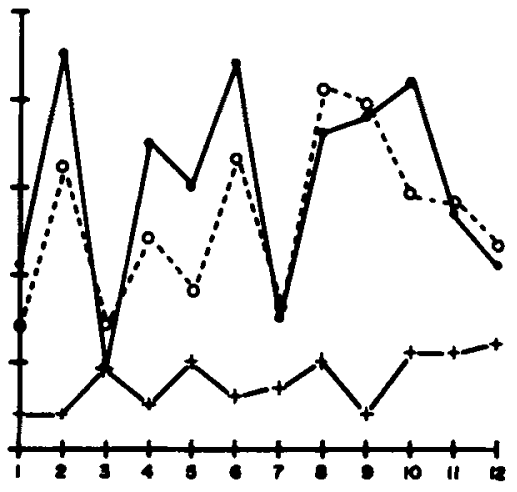

Figure 3. Recognition probabilities $\left(P_{r}\right)$ as a function of the two images, signals, and positions, for $O b-$ servers M.E. and M.M. (A) and (C) present the results for Signal 1; (B) and (D) do so for Signal 2. Bottom curves $(x)$ show average false alarms over the two observers. For signals and positions see Figure 1.

Figure 1. The observer's task was to indicate, by depressing the appropriate button, (a) if the signal had been presented to the right or to the left of the line dividing the image in two halves (a detection response), and (b) whether the signal was or was not the one presented at the beginning of the trial (an identification response). Each signal was embedded 100 times for each image/position combination. On half of these trials, the signal was presented at the onset of the trial as well as embedded in the background (Signal 1-Signal 1 and Signal 2-Signal 2 cases). On the other half of the trials, the signal presented at the beginning of a trial was not the one embedded in the image (Signal 1-Signal 2 and Signal 2-Signal 1 cases). This resulted in a total of 2 signals $\times 100$ trials $\times$ 12 positions $\times 2$ images $=4,800$ trials per observer. These were run in blocks of 480 trials, randomized over the 12 positions, the 4 cases, and the 10 replications for a given image.

\section{RESULTS}

Detection scores were determined from the correct left/right responses, independently of correct or incorrect identification of the signal. These responses, shown in Figure 2, generally demonstrate a high level of performance, since chance behavior, in this case, was $p_{d}=.5$. They also show consistency between the two observers. Recognition probabilities $\left(\boldsymbol{P}_{r}\right.$, Figure 3$)$ were determined from correct detection and correct identification responses over each position. Because of this dual-response paradigm, we can define a "false alarm" as a correct identification but an incorrect detection response, due to the 
(A)

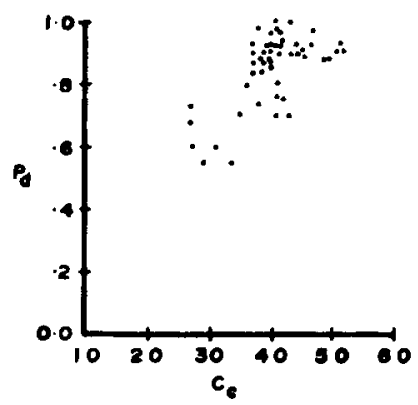

(c)

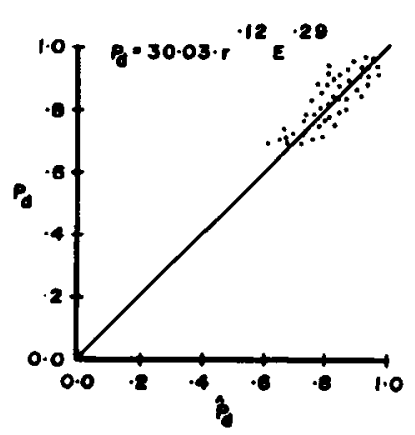

(B)

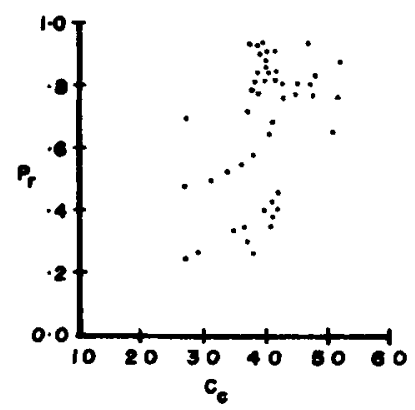

(D)

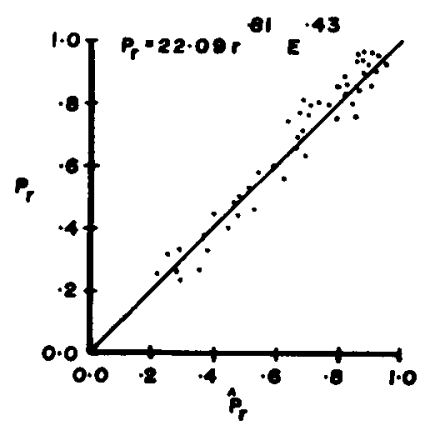

Figure 4. (A) and (B) show detection $\left(P_{d}\right)$ and recognition $\left(P_{r}\right)$ probabilities (averaged over observers) as a function of the cross-correlation between $S$ and $I_{s}$ in each position-over all signals and images $(n=48)$. Here the cross-correlation (CC) values are divided by $100 n^{2}(n=32)$. (C) and (D) correspond to observed and predicted $\hat{P}_{d}$ and $\hat{P}_{r}$ values as a function of the multiple (log) regression of performance with respect to the standardized cross-correlation $(r)$ and energy $(E)$ values.

apparent similarity of other image regions to the signal. These results are shown in Figure 3. Chance recognition performance, in this case, was $P_{r}=.25$.

Figures 4A and 4B show the relationship between detection (a) and recognition (b) as a function of the direct cross-correlation between $S$ and the given $I_{s}$ positions (Figure 1). This relationship is not a significant one, although a trend is discernible. Due to the nonhomogeneous nature of the background images, this result was to be expected, since there are many cases in which a low cross-correlation comes about simply by a lower local space average luminance-although the signal may be quite highly "correlated" "with $I_{s}-$ and vice versa. Due to the relatively low false-alarm and high detection rates, it seems that the observers were quite capable of detecting and recognizing the signals when $I$, was "structurally similar"' to $S$. The problem seems to lie with the inadequacies of the cross-correlation to capture and differentiate this spatial property.

The standardized cross-correlation $\left(r_{12}\right)$ between two images is one representation for "structural similarity," since it is invariant to any linear transformation of contrast or space average luminance, being of the form

$$
-1 \leq r_{12}=\frac{\operatorname{cov}\left(I_{1}, I_{2}\right)}{\sigma_{I_{1}} \cdot \sigma_{I_{2}}} \leq+1 .
$$

Here $r_{12}=0$ signifies independence of the two images. $\sigma_{I_{j}}^{2}$ denotes the variance of image $j$ while cov $\left(I_{1}, I_{2}\right)$ is the covariance between the two. It follows, then, that the cross-correlation function for this experiment can be expressed in terms of $r_{12}$ and energy as follows.

The cross-correlation between $S$ and $I_{s}$ in a given location, in discrete form, is

$$
\begin{aligned}
C_{S, I_{s}} & =\sum_{a, b} S(\alpha, \beta) I_{s}(x+\alpha, y+\beta) \\
& =\operatorname{cov}\left(S, I_{s}\right)+n^{2} \overline{S I}_{s}
\end{aligned}
$$

where

$$
\operatorname{cov}(x, y)=\sum_{x, y}(S(x, y)-\bar{S})\left(I_{s}(x, y)-\bar{I}_{s}\right)
$$

$\bar{S}, \bar{I}_{s}$ correspond to the space average luminances of $S$ and $I_{s}$, respectively.

In our experiment, $\bar{S}=0$ (see Equation 4), and so, via Equation 7, 


$$
C(x, y)=r_{12}(x, y) \cdot \sigma_{s}(x, y) \cdot \sigma_{l_{s}}(x, y) .
$$

Hence, for a given signal $(S)$,

$$
c(x, y)=k \cdot \operatorname{RMS}_{I_{s}} r_{S, I_{s}}(x, y),
$$

where $\mathrm{RMS}_{I_{s}}$ corresponds to the root-mean-squared measure of $I_{s}$ energy, or,

$$
\mathrm{RMS}=\sqrt{\sum_{\alpha, \beta}\left(I_{s}(\alpha, \beta)-\bar{I}_{s}\right)^{2}}
$$

Rather than restrict attention to RMS, we have determined the complete energy of $I_{s}$ in each location by

$$
E_{I_{s}}=\sum_{x, y} I_{s}^{2}(x, y)=\mathrm{RMS}_{I_{s}}^{2}+n \bar{I}_{s}
$$

and computed multiple regressions among detection, recognition scores, and the $r_{S, I}$ and $E_{\mathrm{Is}}$ values at each location (both signals had identical energy values). The results of these log regressions are shown in Figures $4 \mathrm{C}$ and $4 D$, giving multiple regressions of $R=0.72$ for detection and $R=0.84$ for recognition performance (both are significant at the $p<.001$ level). The particular best fitting functions were:

$$
P_{d}=30.3 r^{.12} E^{.29}
$$

and

$$
P_{r}=22.09 r^{.81} E^{.43} \text {. }
$$

As expected, detection performance was more heavily dependent on the $I_{s}$ energy, whereas recognition was critically dependent on the structural similarity-a measure specifically devised to be conceptually independent of the $I_{s}, S$ energy levels (since, as noted before, $r_{S, I_{s}}$ is invariant under linear transformations of both $S$ and $I_{s}$ parameters). This measure overcomes, for example, the fact that with homogeneous or low energy backgrounds, the cross-correlation model predicts low detection and recognition scores (as discussed above), whereas for highenergy uncorrelated (in structure) regions, the crosscorrelation may well be higher than would be expected from the signal to image "structural similarity."

\section{DISCUSSION}

We noted, in the introduction, that models for spatial pattern detection and recognition are of either the "direct" or the "indirect" type. The former models do not propose image decomposition via detectors, whereas the latter do. Both, however, use cross-correlation as the basis for decision, at least for the ideal observer. Within the indirect coding models, there are a variety of approaches, one being the decomposition in terms of specified detectors (spatial frequency channels/filters) and the crosscorrelation of the combined output of such detectors (for example, as via the Minkowski metric; see Wilson \& Gelb, 1984). A second approach maintains that structure may be adequately coded by boundary, luminance differences, or edge information-determined from the outputs of different filters (operators). Again, the outputs of each edge detector would be combined and used as evidence for the existence of $S$ and $I_{s}$.

The question of how many detectors, their independence, and their success in reducing false alarms is the subject of future work. However, it suffices here to show how techniques based on the cross-correlation of "structural similarity" are powerful enough to predict the present recognition data, though not uniquely. The strongest influence on the standardized cross-correlation between $S$ and $I_{s}\left(r_{S, I_{s}}\right.$ in Equations 7-9) is the respective luminance gradients - and hence their edge information. Therefore, it follows that cross-correlations between edgeonly versions of $S$ and $I_{s}$ may be as good predictors of recognition as the formulations in terms of $r_{S, I_{s}}$ and energy $(E)$. For purposes of illustration, we have produced such versions of the images via the following crosscorrelation technique for the extraction of structural similarity alone (see Caelli \& Nagendran, 1985, for more details).

Edges were extracted via the use of a compounded $\nabla^{2}\left(S_{4}\left(G_{\alpha}(F)\right)\right)$ operator. Here $G_{\alpha} \equiv G(\alpha)$, a low-pass Gaussian filter whose bandwidth corresponded to a space constant (1/e decay) of 5 pixels with respect to the $256 \times 256$ image formats. $S_{4}$ corresponds to a "segmentation" operator which resolved the image into four (25th percentile) intensities in order to segment regions of similar luminance. $\nabla^{2}$ corresponded to the digital (finite difference) form of the Laplacian operator:

$$
\nabla^{2} F=\frac{\partial^{2} F}{\partial x^{2}}+\frac{\partial^{2} F}{\partial y^{2}},
$$

which is isotropic, although not necessarily better than the wide range of difference operators available (Rosenfeld \& Kak, 1982). This procedure produces edge contours at the (DC) zero-crossings and at \pm 25 percentile luminances about this value. That is, we have assumed here that zero-crossings are not the only sources of edge information that come from the use of $\nabla^{2} G$, or bandpass filtering. Rather, the visual system is sensitive to a wide range of edge gradient information, and this measure is but one way of capturing more than one type of edge (see Caelli \& Yuzyk, in press, for more information on this issue). We have chosen the particular low-pass aperture ( 5 pixels) to produce bandpass information (via $\nabla^{2} G_{\alpha}$ ) that is sufficiently "edge-like" but yet not too highpass to catch spurious high-frequency false edges.

The images are so reduced to binary ones where nonzero elements code the edge information. As implied in the above discussion, the concept of "edge" is quite an ambiguous term, and we use "edge" here simply to mean the "isoluminance difference" contours that result from the above process. Examples are shown in Figure 5A (top row). The cross-correlation of edge-only images (in this case $S$ and $I_{s}$ ) is particularly simplified by the following.

The discrete form of the cross-correlation function (Equation 1) is:

$$
\hat{C}(x, y)=\sum_{\alpha, \beta} S(\alpha, \beta) . I_{s}(x+\alpha, y+\beta) .
$$


A
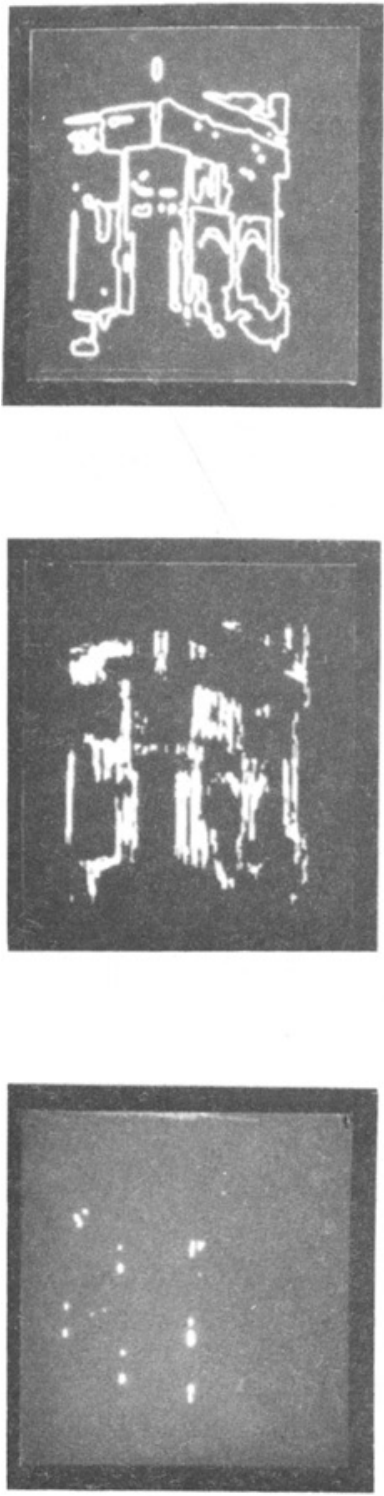

B
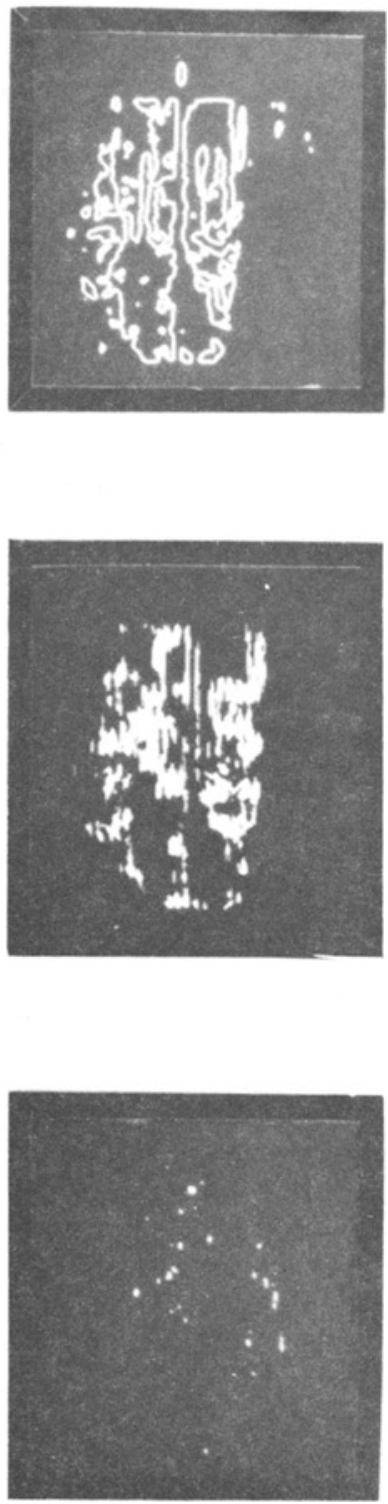

Figure 5. Top row: Edge-only versions of the cafe and Signal 1 images shown in Figure 1 based on the $\nabla^{2}\left(S_{4}(G(F))\right)$ operation described in the text. Second row: Results for the cross-correlation of signal and image edge-only versions. Bottom row: Thresholded versions of Row 2: all values below 128 are deleted (with respect to an 8-bit pixel range).

However, in this case,

$$
S(\alpha, \beta) I_{s}(x+\alpha, y+\beta)=\left\{\begin{array}{c}
1 \text { iff } S(\alpha, \beta) \in E_{s} \\
\text { and } I_{s}(x+\alpha, y+\beta) \in E_{I_{s}} \\
0 \text { elsewhere, }
\end{array}\right.
$$

where $E_{s}, E_{I_{s}}$ correspond to the edge sets of $S$ and $I_{s}$. Hence, Equation 13 reduces to

$$
\hat{C}(x, y)=\sum_{\substack{\alpha, \beta \in E_{\varepsilon^{\prime}} \\ x+\alpha, y+\beta \in E_{l_{s}}}} I_{s}(x+\alpha, y+\beta) . S(\alpha, \beta) .
$$

In other words, $I_{s}$ is added to itself after shifting along the contours defined by $S$. In Row 2 , Figure 5 , the outputs of this process are shown; the bottom row shows a thresholded version $[C(x, y)$ is set to zero when it does not exceed a value of 128 , over an 8-bit pixel range].

We have determined the total response to each signal position ( $I_{s}$, see Figure 1$)$ and compared these with the recognition responses, as shown in Figure 6 . Here the maximum response of the process corresponded to the total number of pixels lying on the signal edge contours. Consequently, we could relate the percentage of $I_{s} / S$ con- 


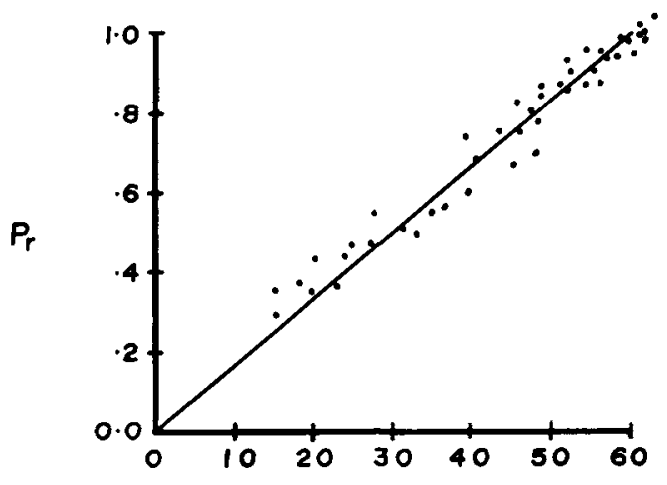

\% 5

Figure 6. The relationship between edge-only cross-correlation of signal and signal-plus-noise with recognition $\left(P_{r}\right)$ scores over all four images and two signals. $\% S$ refers to the percentage ratio of the $S$ and $I_{s}$ cross-correlation to the autocorrelation of $S$ with itself.

tour values to recognition performance. As expected, the fit is almost as good as those for the consideration of full correlation and energy factors. However, we note that this process is also subject to false alarms.

The reasons for considering this extreme formulation for the correlation of structural similarities were because of its apparent efficiency and success in predicting recognition results and because it is not particularly dependent on energy differences over the image. This process is successful in detecting versions of the signal (that is, $I+S$ ) with low energy-so long as the "shape" is sufficiently correlated. Presumably the ideal structural code lies somewhere between this and the complete cross-correlation model.

Here we have not considered the types of spatial decompositions of structural similarity that are likely to occur in such tasks. As already suggested, in conjunction with some form of selectivity or AND gating between the outputs of each detection, false alarms would be reduced. These issues are to be dealt with in a forthcoming paper (Caelli \& Nagendran, 1985).

In conclusion, we have examined the ability of observers to detect and recognize signals embedded in images. In such nonstationary images, we have shown that the matched filter (direct cross-correlation process) does not adequately reflect performance. In contrast, our results indicate that a version of cross-correlation based on the structural similarity between $S$ and $S+I$ reflects the behavior of our observers considerably better. Furthermore, we have presented evidence that even stripping the structural information down to its most elementary form (binary edge-only images) still allows us to predict recognition performance better than the direct energy detection or cross-correlation model. That this latter model works well in homogeneous noise is not surprising. However, performance in this situation would be as well predicted by the approach suggested here.

\section{REFERENCES}

Burgess, A., \& Ghandeharian, H. (1984a). Visual signal detection: I. Ability to use phase information. Journal of the Optical Society of America A, 1, 900-905.

BuRGESs, A., \& GHANDEhaRian, H. (1984b). Visual signal detection II. Signal-location identification. Journal of the Optical Society of America A, 1, 906-910.

CAELLI, T., \& HüBNer, M. (1983). Coding images in the frequency domain: Filter design and energy processing characteristics of the human visual system. IEEE: Transactions on Systems, Man and Cybernetics, SMC-13, 1,018-1,020.

CaElli, T., \& Nagendran, S. (1985). Fast edge-only matching techniques for robot pattern recognition. Manuscript submitted for publication.

Caelli, T., \& YuzYK, J. (in press). On the extraction and alignment of image edges. Spatial Vision.

Daugman, J. (1980). Two-dimensional spectral analysis of cortical receptive field profiles. Vision Research, 20, 847-856.

Daugman, J. (1983). Six formal properties of two-dimensional anisotropic visual filters: Structural principles and frequency/orientation selectivity. IEEE: Transactions on Systems, Man and Cybernetics, SMC-13, 882-887.

JuLEsz, B. (1981). Textons, the elements of texture perception, and their interactions. Nature, 290, 91-97.

Julesz, B. (1985). Towards an axiomatic theory of preattentive vision. In G. M. Edelman, W. E. Gall, \& W. M. Cowan (Eds.), Dynamic aspects of neocortical function. New York: Neurosciences Research Foundation.

Julesz, B., \& Bergan, J. (1983). Textons, the fundamental elements in preattentive vision and perception of textures. Bell System Technical Journal, 62, 1,619-1,643.

Kumar, V., \& Carroll, C. (1984). Loss of optimality in crosscorrelators. Journal of the Optical Society of America A, 1, 392-397.

PAPOUlis, A. (1968). Systems and transforms with applications in optics. New York: McGraw-Hill.

RosenFELD, A., \& KAK, A. (1982). Digital picture processing (Vol. 1) New York: Academic Press.

Wilson, H., GELB, D. (1984). Modified line-element theory for spatial-frequency and width discrimination. Joumal of the Optical Society of America, 73, 1,684-1,690.

(Manuscript received April 24, 1985; revision accepted for publication January 31, 1986.) 